\title{
Reinvestigation of a Critical Reductive Amination Step Leads to an Optimized Protocol for the Synthesis of $\mathbf{N}$-2-hydroxybenzyl- cysteine Peptide Crypto-thioesters.
}

\author{
Skander A. Abboud and Vincent Aucagne* \\ Centre de Biophysique Moléculaire, CNRS UPR 4301, Rue Charles Sadron 45071 Orléans cedex 2, France \\ E-mail: vincent.aucagne@cnrs-orleans.fr
}

\begin{abstract}
We herein report a robust upgraded synthetic protocol for the synthesis of $\mathrm{N}$-Hnb-Cys crypto-thioester peptides, useful building blocks for segment-based chemical protein synthesis through native chemical ligation. We recently observed the formation of an isomeric co-product when using a different solid support than the originally-reported one, thus hampering the general applicability of the methodology. We undertook a systematic study to characterize this compound and identify the parameters favouring its formation. We show here that epimerization from L- to D-cysteine occurred during the key solidsupported reductive amination step. We also observed the formation of imidazolidinones by-products arising from incomplete reduction of the imine. Structural characterization combined with the deciphering of underlying reaction mechanisms allowed us to optimize conditions that abolished the formation of all these sideproducts.
\end{abstract}

\section{Introduction}

The advent of the native chemical ligation (NCL) reaction ${ }^{[1]}$ (scheme 1) opened the realm of proteins to synthetic organic chemists, leading to a new paradigm in protein engineering. NCL substantially pushed away the limits of solid phase peptide synthesis (SPPS, typically limited to a few dozens of amino-acids residues), ${ }^{[2]}$ by providing a simple solution for the assembly of SPPS-synthesized segments. More than 25 years after its discovery, NCL is still, by far, the most widely used approach for chemical protein synthesis ${ }^{[3]}$ and has led to tremendous applications to functional proteins of more than 300 residues. ${ }^{[4]}$

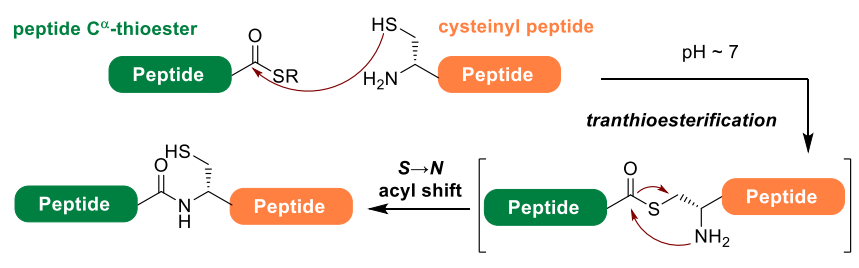

Scheme 1: The native chemical ligation (NCL) reaction

NCL is based on the chemoselective coupling of unprotected peptides bearing a C-terminal thioester and an N-terminal cysteine residue, respectively, to form a native amide bond at a neutral $\mathrm{pH}$ which is optimal for the reaction. Peptide $\mathrm{C}^{\alpha}$-thioesters segments are key building blocks, however the thioester group is not stable towards piperidine treatments used repeatedly in Fmoc-based SPPS for the deprotection of the $N$-Fmoc group. Given that SPPS is conducted from the C- to N-terminus, FmocSPPS is essentially incompatible to the direct synthesis of Cterminal thioesters. A vast number of synthetic methodologies have been explored to circumvent this issue ${ }^{[5]}$ but none is yet generally applicable. Most strategies rely on the introduction of the thioester function after the Fmoc-SPPS elongation of the peptide segment. The most widely used thioester precursors are Liu's hydrazides ${ }^{[6]}$ and Dawson's $\mathrm{N}$-acylureas (Nbz/Me-Nbz) ${ }^{[7]}$ These two C-terminal appendages conveniently allow for the in situ generation of a thioester (either before or during NCL, respectively), classically through thiolysis using a large excess of the arylthiol mercaptophenylacetic acid (MPAA). ${ }^{[8]}$ If these two methodologies are easy to implement and significantly expanded the applicability of NCL-based protein chemical synthesis, they suffer from some limitations ${ }^{[9]}$ and there is still a need for alternative synthetic strategies. ${ }^{[10]}$

Another approach has been increasingly used during the past decade. It exploits the reversible rearrangement through $\mathrm{N}$-to- $S$ acyl shift of a $\beta$ - or $\gamma$-mercapto amide intramolecular thioesterification device, leading a $\beta$ - or $\gamma$-amino thioester, respectively. ${ }^{[11]}$ In most cases, this process necessitates acidic conditions to shift the $N$-S shift equilibrium by protonation of the amine of the rearranged product. Trans-thioesterification with an exogenous thiol and subsequent isolation of the resulting thioester is thus needed prior to NCL. A few $\beta$-mercapto amides, later coined as "crypto-thioesters", ${ }^{[11 \mathrm{k}]}$ are able to rearrange and undergo trans-thioesterification in tandem with NCL, thus considerably simplifying the use of $N \rightarrow S$ shift devices for chemical protein synthesis. A variety of elegant designs of such crypto-thioesters have been proposed. For example, cysteineprolyl esters (CPE) developed by Aimoto ${ }^{[11 f]}$ and later extended to cysteine-prolyl imides (CPI) by Hayashi and Okamoto ${ }^{[11 r]}$ are based on an intramolecular trapping of the amine group. Bissulfanylethylamides (SEA) introduced by Melnyk ${ }^{[11 i]}$ and Liu ${ }^{[11]}$ incorporate a second thiol group that is thought to accelerate the $\mathrm{N}-\mathrm{S}$ shift equilibrium through intramolecular protonation, and Hojo's $N$-alkyl cysteines (NAC) ${ }^{[110]}$ presumably proceed following a similar mechanism, involving in this case the C-terminal Cys carboxylic acid. Otaka's $N$-sulfanylethylanilides (SEAlide ${ }^{[11 k]}$ exploit the destabilization of the amide by an $\mathrm{N}$-aryl substituent and Offer's $\mathrm{C}^{\alpha}$-methyl cysteinamides, ${ }^{[11 \mathrm{n}]}$ an $\alpha$-gem-dialkyl substitution. Despite remarkable applications, all these cryptothioesters show dramatically slower NCL kinetics than preformed alkyl thioesters. An additional drawback of most of these methodologies lies in the difficult coupling of the C-terminal residue of the peptide segment, requiring $\mathrm{N}$-acylation of a sterically-hindered secondary amine. For example, the delicate use of moisture-sensitive coupling agent PyBroP ${ }^{[11 i]}$ and Fmocamino acid fluorides, ${ }^{[11]}$ or long couplings at high temperature using HATU[11e,o] are typically required for acylation of SEA or NAC devices, respectively. Synthetically-demanding preformation of a building block by $\mathrm{N}$-acylation in solution has been shown to be a preferable solution for SEAlides ${ }^{[11 d, k, 12]}$ and NAC. ${ }^{[13]]}$ 
To tackle these limitations, our group reported a few years ago a simple methodology based on an $\mathrm{N}$-(2-hydroxybenzyl) cysteine device ( $N$-Hnb-Cys). ${ }^{[11 p]} \quad N$-Hnb-Cys shows superior $N \rightarrow S$ shift/trans-thioesterification/NCL overall kinetics, owing to an internal catalysis by the phenol group acting as both an $\mathrm{H}$-bond donor ${ }^{[14]}$ binding to the amide oxygen atom and proton donor that stabilizes the $\mathrm{N}-\mathrm{S}$ shift tetrahedral intermediate (scheme 2A). ${ }^{[15]}$ The phenol has an additional effect: it assists the $N$-acylation of the $\mathrm{N}$-Hnb-Cys device through an $\mathrm{O}$-acylation $/ \mathrm{O} \rightarrow \mathrm{N}$-acyl shift mechanism, ${ }^{[16]}$ therefore greatly facilitating the introduction of the C-terminal residue of peptide segments (scheme $2 \mathrm{~B}$ ).
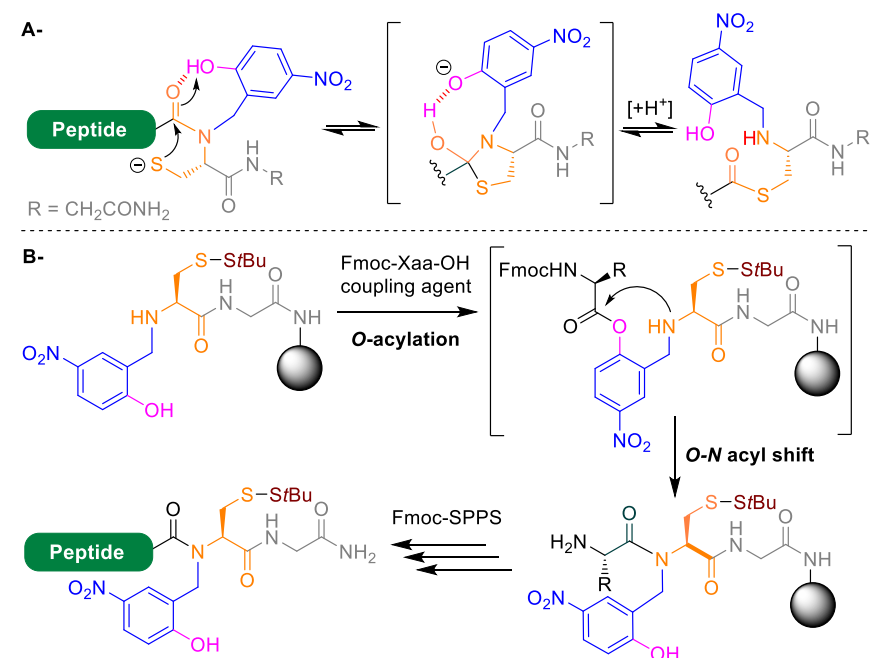

Scheme 2: A) Thioester formation mechanism through $N \rightarrow S$ acyl shift of $N$ Hnb-Cys crypto-thioesters; B) Mechanism of $\mathrm{N}$-acylation of the $\mathrm{Hnb}$-Cys device.

$\mathrm{N}$-Hnb-Cys crypto-thioesters synthesis is straightforward, and can easily be automated on a standard peptide synthesizer using inexpensive building blocks. They have been extensively used by us $^{[17]}$ and others ${ }^{[18]}$ for the synthesis of a wide range of targets, including cyclic peptides, ${ }^{[17 \mathrm{c}]}$ C-terminal-cysteine-containing peptides ${ }^{[17 \mathrm{a}]}$ and other disulfide rich miniproteins, ${ }^{[17 \mathrm{~b}, \mathrm{~d}]}$ as well as a tyrosine kinase receptor domain, ${ }^{[18]}$ illustrating the wide applicability of the method. However, we recently noticed sidereactions when using the initially disclosed synthetic procedure, and decided to take the opportunity for an in-depth study of a key reduction amination step to provide a robust synthetic protocol.

\section{Results and Discussion}

$\mathrm{N}$-Hnb-Cys device synthesis starts with the Fmoc-SPPS coupling of a glycine, then a cysteine bearing a $S$-tert-butylsulfanyl (StBu) protecting group, on a SPPS resin equipped with a Rink amide linker. ${ }^{[19]}$ The Gly residue is introduced to prevent SPPS side reactions like $\beta$-elimination of Cys esters and amide hydrolysis during TFA cleavage of Cys directly linked to a Rink linker. ${ }^{[110]}$ The $\mathrm{StBu}$ protecting group is preferred to the classical trityl to afford the crypto-thioester in a dormant state, unable to rearrange into a hydrolyzable thioester during handling, purification and storage. $\mathrm{StBu}$ is cleaved within a few minutes under classical NCL conditions (including TCEP and MPAA), leading to the active $N$ Hnb-Cys crypto-thioester species. The $\mathrm{N}$-Hnb group is incorporated after Gly and Cys couplings through a two-steps reductive amination reaction to afford the thioesterification device (see fig. 1). The best protocol we identified in our initial report consists in (1) treatment with a dilute acetic acid solution in order to protonate the amine, followed by washings to eliminate excess acid, (2) imine formation through incubation with 10 equiv. of 2 hydroxy-4-nitro benzaldehyde for one hour in 1:1 DMF/MeOH, followed by washings to eliminate excess aldehyde and (3) reduction with a large excess of sodium cyanoborohydride in 9:9:2 $\mathrm{DMF} / \mathrm{MeOH} / \mathrm{AcOH}$. These conditions led to quantitative $\mathrm{Hnb}$ introduction. ${ }^{20]}$

In all our reported applications, ${ }^{[17]} \mathrm{N}$-Hnb-Cys crypto-thioesters were synthesized on an amino-Tentagel $\mathrm{R}$ resin, a PEG-grafted polystyrene. Recently, during attempts to use ChemMatrix, a PEG-based polymer known for its superior properties for the synthesis of long or "difficult" peptide sequences, ${ }^{[21]}$ we observed a satellite to the major peak: cleavage of the resin after the deprotection of the Fmoc group of the first residue of the peptide segment, an $\mathrm{O}$-tBu-serine in this case, similarly led to a mixture of the expected $\mathrm{H}$-Ser-(Hnb)Cys(StBu)-Gly- $\mathrm{NH}_{2}(4)$ together with a significant side-product (5) (8-15\%) showing the same $\mathrm{m} / \mathrm{z}$ in MS analysis (fig. 1B), indicating an isomerization during either the $\mathrm{N}$-Hnb-Cys device synthesis or the Ser coupling
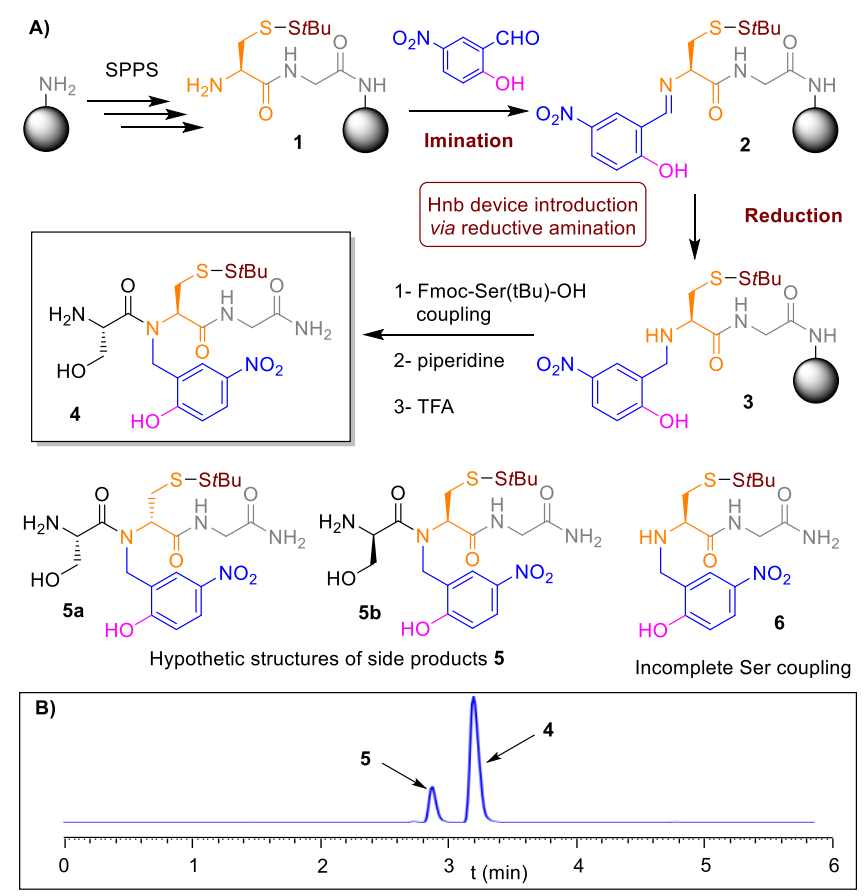

Figure 1: A) Synthesis of the $\mathrm{N}$-Hnb-Cys thioesterification device through solid phase reductive amination (conditions: see text). B) HPLC chromatogram ( $\lambda=$ $320 \mathrm{~nm}$ ) obtained when using a ChemMatrix resin, showing a satellite to the major peak after serine coupling and TFA cleavage.

This result was somehow surprising, as we previously reported the synthesis of $\mathbf{4}$ on Tentagel without observing any significant impurities. ${ }^{[23]}$ This prompted us to investigate the influence of the polymer support on the generation of its isomer $\mathbf{5}$, since resins do not behave as inert carriers but rather act as a co-solvents that can affect various parameters of a chemical reaction such as yield, purity, rate and parasite reactions. ${ }^{[24]} A$ resin screening study (table 1) showed that the amount of side-product is indeed highly dependent on the nature of the polymer support: the poly-lysinebased resin Spheritide ${ }^{[25]}$ and ChemMatrix gave the worst results, followed by the PEG-grafted polystyrene resin Novagel ${ }^{[26]}$ and polystyrene (see supporting information, scheme $p$ S4) for polymers structures). We also observed the formation of $\mathbf{5}$-albeit in a lower extent- when using Tentagel, in contradiction with our initial report. Several repetitions of the later experiment led to 
similar results, even if the amount of $\mathbf{5}$ slightly varied upon batches $(5-8 \%)$.

Table 1. Resin effect on side product 5 formation during the synthesis of 4 . $^{\text {[a] }}$

\begin{tabular}{llcll}
\hline Entry & Resin & Loading $(\mathrm{mmol} / \mathrm{g})$ & $\mathbf{4}(\%)^{[\mathrm{b}]}$ & $\mathbf{5}(\%)^{[\mathrm{b}]}$ \\
\hline 1 & ChemMatrix & 0.54 & 89 & 11 \\
2 & Tentagel R & 0.16 & 93 & 7 \\
3 & Polystyrene & 0.89 & 91 & 9 \\
4 & Spheritide & 0.15 & 86 & 14 \\
5 & Novagel & 0.77 & 90 & 10 \\
\hline
\end{tabular}

[a] Reaction conditions ${ }^{[110]}$ : imination with 10 equiv. aldehyde, 1 equiv. $\mathrm{AcOH}$ in 1:1 DMF/MeOH, $12.5 \mathrm{mM}$ peptidyl resin, $\mathrm{RT}$; reduction with 20 equiv. $\mathrm{NaBH}_{3} \mathrm{CN}$ in 9:9:2 DMF/MeOH/AcOH, $12.5 \mathrm{mM}$ peptidyl resin, $\mathrm{RT}$; [b] Relative rates determined by integration of HPLC peaks at $\lambda=320 \mathrm{~nm}\left(\lambda_{\max } \mathrm{Hnb}\right)$.

We hypothesized that one of the chiral amino acid residues epimerized during the synthesis, either Cys (leading to compound $\mathbf{5 a}$ ) or Ser (compound $\mathbf{5 b}$ ), and we decided to carry out a systematic study to understand and minimize this side reaction, focusing on the ChemMatrix resin that gave particularly high amounts of $\mathbf{5}$. At first, we assumed that the serine residue could have likely epimerized during its coupling. Indeed, Ser is known to be one of the proteogenic amino acids most prone to epimerization upon coupling, ${ }^{[27]}$ after $\mathrm{Cys}^{[28]}$ and His. ${ }^{[29]} \mathrm{We}$ thought that the $\mathrm{O} \rightarrow \mathrm{N}$ shift mechanism assumed for Hnb-Cys $\mathrm{N}$ acylation could potentially have enhanced this propensity.

Table 2. Effectiveness of different coupling conditions for Ser coupling on $3^{[a]}$

\begin{tabular}{lcclccc}
\hline $\begin{array}{l}\text { Entry } \\
\text { agent }\end{array}$ & $\begin{array}{c}\text { Coupling } \\
1\end{array}$ & Base & Solvent & $\begin{array}{c}\mathbf{4}+\mathbf{5} \\
(\%)^{[\mathrm{b}]}\end{array}$ & $\begin{array}{c}\mathbf{4}: \mathbf{5} \\
\text { ratio }^{[\mathrm{b}]}\end{array}$ & $\begin{array}{l}\mathbf{6} \\
(\%)^{[\mathrm{b}]}\end{array}$ \\
\hline 2 & HATU & DIEA & DMF & 100 & $89: 11$ & - \\
3 & DIEA & DMF & 100 & $90: 10$ & - \\
4 & DIC/Oxyma & - & DMF & 100 & $90: 10$ & - \\
5 & HCTU & NMM & DMF & 93 & $85: 15$ & 7 \\
6 & HCTU & DIEA & DCM/DMF & 78 & $91: 9$ & 22 \\
& & & & 100 & $90: 10$ & - \\
& & & & & &
\end{tabular}

[a] $18 \mathrm{~h}$ coupling, $10 \mathrm{mM}$ peptidyl resin, 10 equiv. Fmoc-Ser $(\mathrm{tBu})-\mathrm{OH}, 9.5$ equiv. coupling agent, 20 equiv. base, RT ; [b] Relative rates determined by integration of HPLC peaks at $\lambda=320 \mathrm{~nm}$.

Therefore, we tried to optimize Ser coupling, focusing on reactions conditions having been described to reduce epimerization in specific cases (table 2). We did not observe any significant improvement in the 4 / 5 ratio $(9-11 \% 5)$, and even a slight increase when using $\mathrm{N}$-methyl-morpholine (NMM) as the base (entry 5). We also monitored the completion of the coupling: the reaction went to completion in most cases except when using NMM, or 1:1 DCM/DMF as solvent (entries 5 and 6 , respectively, observation of the non-acylated compound 6 ).

Puzzled by these results, the next hypothesis we explored was Cys(StBu) epimerization, either as the sole source of isomer formation, or in addition to Ser epimerization. For this purpose we coupled an extra Val residue after Ser, in order to add a third chiral centre and obtain diastereoisomers discernible by classical achiral RP-HPLC, instead of the co-eluted enantiomers $\mathbf{5 a}$ and $\mathbf{5 b}$. This led to a mixture of four HPLC peaks showing the same $\mathrm{m} / \mathrm{z}$, in a $89.2: 9: 1.6: 0.2$ ratio (fig. $2 \mathrm{~B}$ ). These peaks were supposed to correspond to all four diastereoisomers 7a-d arising from Cys and/or Ser epimerization, the major peak being obviously the nonepimerized product $\mathbf{7 a}$. In order to unambiguously attribute the three remaining peaks to putative structures $\mathbf{7 b}$-d, we generated three HPLC standards corresponding to Cys epimerization (7b), Ser epimerization (7c) and both Ser and Cys epimerization (7d). In the case of $\mathbf{7 b}$ and $\mathbf{7 d}$, we synthesized their enantiomeric counterpart $\mathbf{7 e}$ and $\mathbf{7 f}$, respectively, as the later compounds were less synthetically demanding while being equivalent in achiral HPLC (fig. 2A).

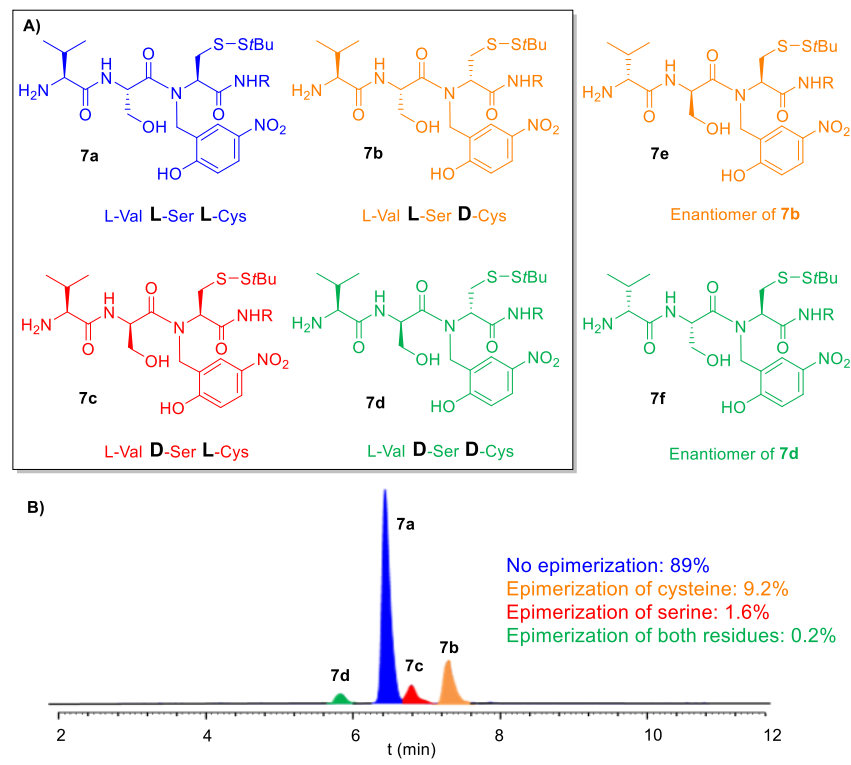

Figure 2: A) Structures of the four diastereoisomer products 7a-d and the HPLC standards 7e and 7f. $\mathrm{R}=\mathrm{CH}_{2} \mathrm{CONH}_{2}$. B) HPLC Chromatogram $(\lambda=320 \mathrm{~nm})$ of crude mixture 7 . \% determined by HPLC peak integration.

As expected, this led in each case to a major peak accompanied with three minor ones. Co-injection of the four crude mixtures led to four well-separated HPLC peaks (SI, fig S9). This allowed us to identify the peaks in the initial chromatogram: as anticipated, the most important epimerization corresponds to D-cysteine $(\mathbf{7 b}+\mathbf{7 d}$ : $9.4 \%)$, even if a small amount of D-serine $(7 \mathrm{c}+7 \mathrm{~d}: 1.8 \%)$ was also detected. (fig. 2B).

Having clearly established that it is the cysteine residue that mostly epimerizes, we were quite relieved about the efficiency of our previously-described protocol for applications to chemical protein synthesis: indeed, this meant that the epimerized residue is not be present in ligation products as a consequence of the expulsion of the $\mathrm{N}-\mathrm{Hnb}-\mathrm{D} / \mathrm{L}-\mathrm{Cys}$ thioesterification device during NCL. $\left.{ }^{[30]}\right]$ However, D-Cys formation complicates the purification of the crypto-thioester segments and is deleterious in terms of isolated yields and product purity. We thus decided to pursue a systematic investigation to determine the origin of Cys epimerization. We started by checking the enantiomeric purity of our commercial cysteine building block, through formation of diastereoisomers by coupling with D- and L-versions of a chiral amine. No D-Cys isomer was detected (supporting information, $p$ S14). Next, in order to assess if epimerization happens during the coupling of the cysteine residue, we synthetized the tripeptide $\mathrm{H}$ Ser-Cys(StBu)-Gly- $\mathrm{NH}_{2}$ (8) devoid of Hnb group, together with an 
HPLC standard corresponding to the epimerized product (supporting information, p S15). We did not detect any D-Cys, which means that the epimerization occurs during the introduction of the Hnb group.

We hypothesized that Cys epimerization could likely arise from a tautomeric equilibrium between aldimine 2 and a rearranged achiral ketimine 9 (scheme 5) prior to reduction into amine $\mathbf{3}$. Such imine tautomerism has been widely investigated in synthetic organic chemistry, ${ }^{[31]}$ and can be catalysed by strong bases ${ }^{[32]}$ acids $^{[33]}$ metals ${ }^{[34]}$ or high temperatures. ${ }^{[35]}$ This rearrangement has been exploited in protein and peptide chemistry for the bioinspired conversion of $\mathrm{N}$-terminal amines to ketones in aqueous media, through a ketimine intermediate which is further hydrolyzed. ${ }^{[36]}$ Careful re-examination of the literature in the solid phase synthesis field told us that this side reaction has also been observed during the grafting of amino acid derivatives on a backbone amide linker-functionalized resin through reductive amination, and was even intentionally used for the complete racemization of chiral amino acids. ${ }^{[33]}$

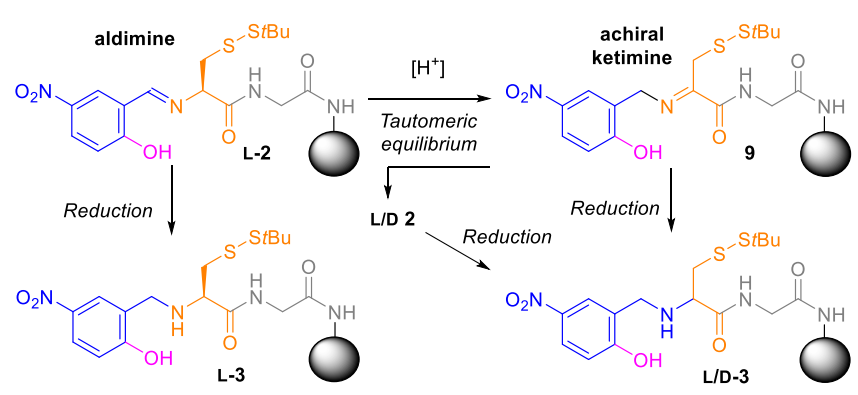

Scheme 3: Putative epimerization mechanism through amine tautomerism.

To probe the imine tautomerism hypothesis, we varied the imination time prior to treatment with sodium cyanoborohydride, followed by coupling of Fmoc-Ser(tBu)-OH. Consistently, prolongation from one to four hours dramatically enhanced the formation of the side-product 5 , (table 3 , entries 1 and 2 , respectively). Reduction of the imination time to $5 \mathrm{~min}$ only led to a moderate decrease of the formation of 5 (table 3 , entry 3 ): this could be explained by a slow reduction of the imine by $\mathrm{NaBH}_{3} \mathrm{CN}$, leaving enough time for the deleterious tautomeric equilibrium to proceed during the reduction step. An overnight treatment of the reduced compound (3) under the same reduction conditions did not change the $4: 5$ ratio, confirming that epimerization does not happen after reduction of the imine.

Table 3. Effect of the variation of imination reaction time on epimerization.

\begin{tabular}{lccccc}
\hline Entry & $\begin{array}{c}\text { Reducing } \\
\text { agent }\end{array}$ & $\begin{array}{c}\text { Imination } \\
\text { time }\end{array}$ & $\begin{array}{c}\text { Reduction } \\
\text { time }\end{array}$ & $\mathbf{4}(\%)^{[\mathrm{c}]}$ & $\mathbf{5}(\%){ }^{[\mathrm{c}]}$ \\
\hline $1^{[\mathrm{d}]}$ & $\mathrm{NaBH}_{3} \mathrm{CN}$ & $1 \mathrm{~h}$ & $1 \mathrm{~h}$ & 89 & 11 \\
2 & $\mathrm{NaBH}_{3} \mathrm{CN}$ & $4 \mathrm{~h}$ & $1 \mathrm{~h}$ & 80 & 20 \\
3 & $\mathrm{NaBH}_{3} \mathrm{CN}$ & $5 \mathrm{~min}$ & $1 \mathrm{~h}$ & 92 & 8
\end{tabular}

[a] Imination conditions : 10 equiv. aldehyde, 1 equiv. $\mathrm{AcOH}, 1: 1 \mathrm{DMF} / \mathrm{MeOH}$, $12.5 \mathrm{mM}$ peptidyl resin, RT; [b] Reduction conditions : 9:9:2 DMF/MeOH/AcOH, $12.5 \mathrm{mM}$ peptidyl resin, RT; [c] Relative rates determined by HPLC peak integration at $\lambda=320 \mathrm{~nm}$. [d]: initial conditions from reference [11o].
If things started to be clearer on the rationale behind the formation of byproduct 5 , we remained intrigued by the low reproducibility of our results (present and past experiments ${ }^{[110]}$ ). We questioned if the relative notion of "room" temperature, or other biases upcoming from the conduction of the reaction on a solid support (efficiency of the washing steps, actual final concentrations etc.) could be responsible for this lack of reproducibility. High temperatures favor the formation of 5 : up to $32 \%$ when the reaction is performed at $60^{\circ} \mathrm{C}$, and only $8 \%$ at $4^{\circ} \mathrm{C}$ (supporting information, table S2). Epimerization depends on concentration: the more the reaction is diluted the more the rate of the epimer increases (supporting information, table S3). Moreover, the amount of acetic acid used during the imine formation step also modulates the formation of 5 (table 4). Use of a large excess (10\% $v / v, 140$ equiv., entry 4) dramatically increased epimerization, while a lower concentration ( $1 \%$, entry 3 ) slightly reduced it, as compared with our initial conditions where no extra $\mathrm{AcOH}$ was added in addition to the 1 equiv. acetate counter anion arising from prior amine protonation (entry 2).

Table 4. Variation of acetic acetic acid amount during imination reaction. ${ }^{[a]}$

\begin{tabular}{llll}
\hline Entry & Additives & $\mathbf{4}(\%)$ & $\mathbf{5}(\%)^{[\mathrm{b}]}-$ \\
\hline 1 & No additive & 89 & 11 \\
2 & 1 equiv. AcOH & 89 & 11 \\
3 & 14 equiv. AcOH $(1 \% v / v)$ & 91 & 9 \\
\hline
\end{tabular}

[a] Reaction conditions: Imination in 1:1 DMF/MeOH for $1 \mathrm{~h}$ at RT, $12.5 \mathrm{mM}$ peptidyl resin; reduction in DMF/MeOH/AcOH (9:9:2) for $1 \mathrm{~h}$ at $\mathrm{RT}$. [b] Relative rates determined by integration of HPLC peaks at $\lambda=320 \mathrm{~nm}$.

We believe that this high overall sensitivity to reaction conditions is likely responsible for the divergence between our initial report and this work. This prompted us to start a re-optimization of the reductive amination, willing to establish a highly reproducible protocol.

Having demonstrated that imine 2 does undergo epimerization, we focused on reducing its lifetime during the reductive amination process. We thus re-investigated one-pot imination/reduction, followed by Ser coupling, with the goal to minimize the formation of $\mathbf{5}$ as well as of non- or bis-alkylated products observed in our initial study when trying different conditions to optimize the reductive amination step. We explored the use of sodium cyanoborohydride, in addition to pyridine borane and sodium triacetoxyborohydride, two other classical reagents for solutionphase one-pot reductive amination ${ }^{[37]}$ (table 5). ${ }^{[38]}$ The one pot process greatly minimized epimer 5 formation, down to a 2\% rate very close to epimerization accounting from the Ser residue (1.8\%, fig. 2). However, significant amounts (2-17\%) of nonalkylated compound $\mathbf{8}$ were also produced, in line with our initial study. Surprisingly, use of sodium tri-acetoxyborohydride led to the formation of four different peaks (10a-d, entry 8) which masses correspond to [M-2 Da] of the desired product, and no traces of either $\mathbf{4}$ or $\mathbf{5}$. After re-investigation of the LC-MS chromatograms of all crude products, small amounts of the same new byproducts $\mathbf{1 0}$ were also observed in most other one-pot reductive aminations (entries 1-7), while being absent when using our initial protocol. Use of pyridine borane in $\mathrm{DMF} / \mathrm{MeOH}$ gave satisfactory results in terms of epimer 5 and [M-2 Da] 10 
byproducts formation, albeit accompanied with the generation of non-alkylated compound (entry 4), that can be minimized using prolonged reaction time (entry 7 ). If the latter conditions proved to be highly reproducible and relatively well suited for manual introduction of the Hnb group, it was difficult to adapt to an automated version, due to the observed instability of the reducing agent upon storage in solution for a few hours.

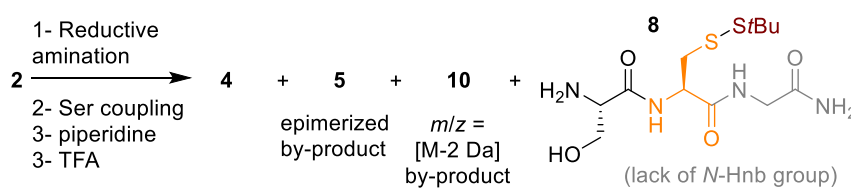

Scheme 4: Mixture of products obtained after one pot reductive amination

Table 5. One pot reductive amination. ${ }^{[a]}$

\begin{tabular}{|c|c|c|c|c|c|c|}
\hline Entry & $\begin{array}{l}\text { Reducing } \\
\text { agent }\end{array}$ & Solvent & $\begin{array}{l}4^{[b]} \\
(\%)\end{array}$ & $\begin{array}{l}5^{[b]} \\
(\%)\end{array}$ & $\begin{array}{l}10^{[\mathrm{b}]} \\
(\%)\end{array}$ & $\begin{array}{l}8^{[c]} \\
(\%)\end{array}$ \\
\hline $1^{[d]}$ & $\mathrm{NaBH}_{3} \mathrm{CN}$ & $\mathrm{DMF} / \mathrm{MeOH}$ & 84.3 & 2.2 & 4.2 & 9.3 \\
\hline 2 & $\mathrm{NaBH}_{3} \mathrm{CN}$ & $\mathrm{MeOH}$ & 94.6 & 1.9 & 0.6 & 2.9 \\
\hline 3 & $\mathrm{NaBH}_{3} \mathrm{CN}$ & THF & 84 & 3.8 & 8.7 & 3.5 \\
\hline $4^{[d]}$ & Pyridine $\cdot \mathrm{BH}_{3}$ & $\mathrm{DMF} / \mathrm{MeOH}$ & 86.4 & 1.8 & $<0.1$ & 11.8 \\
\hline $5^{[\mathrm{d}, \mathrm{e}]}$ & Pyridine $\cdot \mathrm{BH}_{3}$ & $\mathrm{DMF} / \mathrm{MeOH}$ & 95.5 & 2 & $<0.1$ & 2.5 \\
\hline 6 & Pyridine $\cdot \mathrm{BH}_{3}$ & $\mathrm{MeOH}$ & 80.5 & 2.1 & 0.3 & 17.1 \\
\hline 7 & Pyridine $\cdot \mathrm{BH}_{3}$ & THF & 75.4 & 2.4 & 17.7 & 4.5 \\
\hline $8^{[\mathrm{ft}]}$ & $\mathrm{NaBH}(\mathrm{OAc})_{3}$ & THF/MeOH & 0 & 0 & 100 & 0 \\
\hline
\end{tabular}

[a] 5 min reactions using 10 equiv. aldehyde, 20 equiv. reducing agent, $1 \%$ $\mathrm{AcOH}$ as additive, $\mathrm{RT}, 12.5 \mathrm{mM}$ peptidyl resin; [b] Relative rates determined by HPLC peak integration at $\lambda=320 \mathrm{~nm}$; [c] Relative rate determined by coupling of an extra Trp residue and integration of HPLC peaks at $\lambda=280 \mathrm{~nm}$ (see supporting information $\mathrm{p} \mathrm{S45);} \mathrm{[d]} \mathrm{1:1} \mathrm{DMF/MeOH} \mathrm{ratio;} \mathrm{[e]} 20$ min reaction; [f] 1:1 THF/MeOH ratio.

Prior to further optimization of a synthetic protocol compatible with routine automation on a peptide synthesizer, we wanted to decipher the formation of the [M-2 Da] byproducts 10 . Considering that this mass shift is consistent with compounds that would have not been reduced, we tried imine formation followed by overnight Ser coupling, without treatment by any reducing agent. This led to a chromatogram essentially identical to the one obtained with sodium triacetoxyborohydride, demonstrating that $\mathbf{1 0}$ indeed arose from incomplete reduction of the imine. We next wanted to characterize the four products $\mathbf{1 0 a - d}$ by NMR in order to elucidate their chemical structure. Separation of the four peaks proved to be very challenging, but we succeeded in isolating small quantities of the three compounds $10 a-c$, as well as $\mathbf{1 0 d}$ in mixture with $\mathbf{1 0 c}$. Disappointingly, extensive NMR characterization was not feasible due to the presence of two or more different sets of NMR peaks in each purified compounds, probably arising from conformers in slow exchange, which considerably complicated the spectra. ${ }^{[39]}$ However, combination of $1 \mathrm{D}$ and $2 \mathrm{D}{ }^{1} \mathrm{H},{ }^{13} \mathrm{C}$ and ${ }^{15} \mathrm{~N}$ experiments (reported in details in the supporting information, p S27-S41) allowed us to elaborate a solid hypothesis for the structures of 10a-d. In a few words, we observed for the four products the disappearance of the benzylic $\mathrm{CH}_{2}$ signals observed in both $\mathbf{4}$ and $\mathbf{5}$, accompanied with the apparition of new $\mathrm{CH}$ singlets at 6.0-6.7 ppm. This suggested the formation of compounds C-disubstituted in $\alpha$ of the nitrophenol ring. ${ }^{[40]}$ Moreover, ${ }^{1} \mathrm{H}-{ }^{15} \mathrm{~N}$ HSQC spectra of 10a-d showed the disappearance of the $\mathrm{NH}$ amide signal of the Cys-Gly peptide bond, while glycine C-terminal $\mathrm{CONH}_{2}$ and serine $\mathrm{NH}_{3}{ }^{+}$(TFA salt) remained essentially unchanged: this suggested an $\mathrm{N}$-substitution of the Cys-Gly amide nitrogen. We consequently deducted the probable $\mathrm{N}$-acyl imidazolidinone structures shown in scheme 5 . This hypothesis was further strengthened by comparison with literature NMR data for related compounds: ${ }^{1} \mathrm{H}$ and ${ }^{13} \mathrm{C}$ chemical shifts of the newly formed $\mathrm{CH}$ signals in 10a-d are consistent with those reported for structurally-related $\mathrm{N}$-acylimidazolidinones (see supporting information, p S42). ${ }^{[41]}$

From a mechanistic point of view, these five-membered rings could be obtained through two different known pathways (scheme 5 ). In the first one, a highly reactive $\mathrm{N}$-acyliminium would be formed from the coupling of serine with the nonreduced imine, followed by intramolecular trapping with the Cys-Gly amide nitrogen acting as a nucleophile (green pathway). Very similar reaction were reported in the literature using T3P as a strong carboxylic acid activator. ${ }^{[42]}$ In our case, overnight reaction using HCTU could be sufficient to promote coupling with the poorly nucleophilic imine, and assistance of the Hnb phenol group for imine $\mathrm{N}$-acylation, similar to the $\mathrm{O}$-acylation/O $\rightarrow \mathrm{N}$-acyl shift mechanism during amine $\mathrm{N}$-acylation of the reduced compound 3, can also be advocated. In a second plausible hypothesis, nucleophilic attack of the Cys-Gly amide nitrogen on the non-reduced imine would generate imidazolidinone 11, followed by serine coupling (red pathway). Related reactions were recently exploited by Francis and Raj groups for protein immobilization on polymeric supports and for the synthesis of conformationally rigid macrocycles through chemo- and diastereoselective cyclization ("CyClick chemistry") respectively. ${ }^{[43]}$

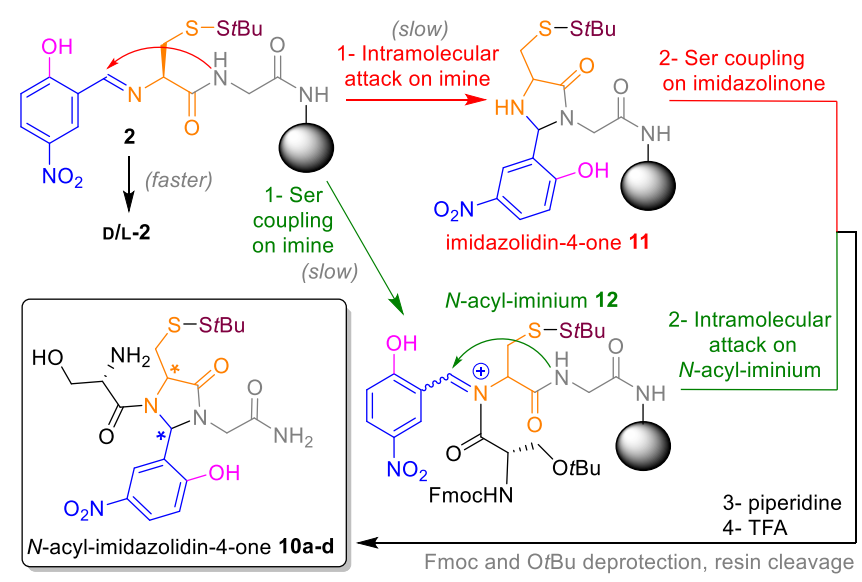

Scheme 5: Mechanistic hypotheses for imidazolidinones 10a-d formation.

Note that, in both hypotheses, imine $\mathbf{2}$ is expected to have a long lifespan during the overnight Ser coupling, thus being consistent with a massive epimerization of Cys through aldimine-ketimine tautomerism prior to imidazolidinone formation. Moreover, addition of the Cys-Gly amide nitrogen on the $\mathrm{N}$-acyliminium or imine is likely to proceed in a poorlydiastereoselective fashion, affording epimeric products at the 
benzylic position. These two considerations are fully consistent with the formation of comparable amounts of each

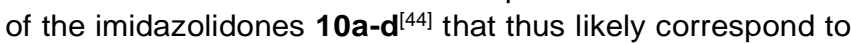
the four possible diastereoisomeric structures depicted in scheme $5 .{ }^{[45]}$

Having elucidated the structure of all byproducts (5a-b, 10ad) together with drawing up plausible hypotheses that explain their formation, we turned back to our initial goal of protocol optimization. Our findings taught us that the lifespan of imine 2 should be kept as short as possible. In consequence, we designed a simple protocol based on a short imination time, combined with very fast reduction of the imine. Considering that we observed slightly higher formation of 5 when using either no acid additive or a large excess (see above, table 4), we conducted the imination through a $5 \mathrm{~min}$ treatment with a tenfold excess of 5-hvdroxv-2-

supplemented with $1 \% \mathrm{AcOH}$. After prompt resin washes, we treated the imine resin 2 with a large excess of sodium borohydride $\left(\mathrm{NaBH}_{4}\right)$ in DMF. This reducing agent is stronger than sodium cyanoborohydride and in contrast with the latter, $\mathrm{NaBH}_{4}$ is known to rapidly reduce imines without requiring iminium formation through protonation. It is also known to react with acids and methanol, reason why we used neat DMF. Very gratifyingly, these conditions resulted in (1) no observation of 10a-d upcoming from incomplete reduction, (2) no formation of non- or bis-alkylated products and (3) only $2 \%$ epimerization, thus very close to the $1.8 \% \mathrm{D}$-Ser formation during coupling (fig. 2). ${ }^{[46]}$ On top of that, we obtained equivalent results after keeping the $\mathrm{NaBH}_{4}$ solution in DMF for at least one day at room temperature, an ideal feature for automated $\mathrm{N}$-Hnb-Cys crypto-thioesters preparation.

Table 6. Two-steps $\mathrm{NaBH}_{4}$ reductive amination. [a]

\begin{tabular}{lcccccc}
\hline $\begin{array}{c}\text { Entry } \\
\text { time }\end{array}$ & $\begin{array}{c}\text { Imination } \\
\text { time }\end{array}$ & $\begin{array}{c}\mathbf{4}^{[\mathrm{b}]} \\
(\%)\end{array}$ & $\begin{array}{c}\mathbf{5}^{[\mathrm{b}]} \\
(\%)\end{array}$ & $\begin{array}{c}\mathbf{1 0}^{[\mathrm{b}]} \\
(\%)\end{array}$ & $\begin{array}{c}\mathbf{8}^{[\mathrm{c}]} \\
(\%)\end{array}$ \\
\hline 1 & $5 \mathrm{~min}$ & $20 \mathrm{~min}$ & 98 & 2 & $<0.1$ & $<0.1$
\end{tabular}

[a] Reaction conditions : Imination with 20 equiv. aldehyde in DMF/MeOH 1:1 $+1 \% \mathrm{AcOH}, \mathrm{RT}, 12.5 \mathrm{mM}$ peptidyl resin; reduction with 20 equiv. $\mathrm{NaBH}_{4}$ in $\mathrm{DMF}$ RT, $12,5 \mathrm{mM}$ peptidyl resin. [b] Relative rates determined by HPLC peak integration at $\lambda=320 \mathrm{~nm}$. [c] Relative rate determined by coupling of an extra Trp residue and integration of HPLC peaks at $\lambda=280 \mathrm{~nm}$ (see supporting information $\mathrm{p}$ S45).

With such a gold standard protocol for reductive amination in hands, we wanted to assess the actual Cys vs Ser epimerization under these conditions, and thus coupled an extra Val for diastereoisomer formation. We were pleased to observe that $\mathrm{Cys}(\mathrm{StBu})$ epimerization was reduced to as low as $0.3 \%$ (supporting information, supplementary figure $\mathrm{S} 10 \mathrm{~b})$. The remaining $1.7 \%$ corresponds to Ser epimerization (product $\mathbf{7 b}$ ) during its coupling. We finally reinvestigated the coupling conditions of the Ser residue on the $\mathrm{N}$-Hnb-Cys(StBu) device (3), DIC/Oxyma giving significantly improved results $(0.6 \%$ Ser epimerization, see supplementary information figure $\mathrm{S} 11$ and table $\mathrm{S6}$ ). Gratifyingly, these conditions also gave excellent results for the coupling of Cys(Trt) and $\mathrm{His}(\mathrm{Trt}) \quad(0.8 \%$ and $0.2 \%$ epimerization, respectively, see supporting information tables S4 and S5).

The new reductive amination protocol was finally challenged with the automated synthesis of two different peptides. The model peptide crypto-thioester H-Leu-Tyr-Arg-Ala-Ala$(\mathrm{Hnb}) \mathrm{Cys}(\mathrm{StBu})-\mathrm{Gly}-\mathrm{NH}_{2}$ (S23) was synthesized on Tentagel, ChemMatrix and Rink-MBHA-polystyrene resins (supporting information fig. S48) giving clean products devoid of any detectable above-mentioned side-products arising from epimerization or incomplete reduction. Synthesis of a very long 62 amino acids peptide cryptothioester, the sequence of which derives from the human mucin $\mathrm{MUC1}^{[47]}$ (supporting information, fig. S49, compound S24) also gave excellent results, fully validating our optimized protocol.

\section{Conclusion}

In conclusion, an in-depth reinvestigation of previously described protocols for $\mathrm{N}$-Hnb-Cys peptide crypto-thioesters synthesis led to the identification of a variety of deleterious by-products. The major one corresponds to the epimerization of the Cys residue during solid-supported reductive amination. Fine understanding of the structures of the byproducts combined with the deciphering of their mechanism of formation allowed to optimize a highly-reproducible synthetic protocol leading to clean peptide crypto-thioester segments. In addition to providing a gold standard automatable protocol for the preparation of $\mathrm{N}$-Hnb-Cys peptide crypto-thioesters for applications to the chemical synthesis of proteins, the findings herein reported could be significant for the synthesis of $\mathrm{N}$-alkylated chiral amino acids or peptide through reductive amination, either in solution or on solid phase. ${ }^{[48]}$

\section{Experimental Section}

All experimental protocols and compounds characterization are detailed in the supporting information file.

\section{Recommended protocol for the synthesis of $\mathbf{N}$-Hnb-Cys crypto- thioesters.}

Reductive amination: $25 \mu \mathrm{mol}$ of a $\mathrm{H}$-Cys(StBu)Gly-Rink-resin synthesized through standard Fmoc-SPPS was washed with a 1:1 DMF/MeOH mixture (4 × $3 \mathrm{~mL}$ for $30 \mathrm{~s})$. 2-Hydroxy-5-nitrobenzaldehyde (42 mg, 0.25 mmol, 10 equiv.) in $2 \mathrm{~mL}$ 44.5:44.5:1 DMF/MeOH/AcOH (125 $\mathrm{mM}$ aldehyde concentration) was then added, and the reaction vessel was stirred for $5 \mathrm{~min}$ at room temperature. Solvents were drained and the resin was washed with 1:1 DMF/MeOH $(3 \times 3 \mathrm{~mL}$ for $5 \mathrm{~s})$ then DMF $(3 \times 3 \mathrm{~mL}$ for $5 \mathrm{~s}$ ). Without delay, a solution of sodium borohydride $(19 \mathrm{mg}, 0.5 \mathrm{mmol}$, 20 equiv.) in $2 \mathrm{~mL}$ DMF ( $250 \mathrm{mM}$ borohydride concentration) was added and the reaction vessel was stirred for $20 \mathrm{~min}$ at room temperature. Solvents were drained and the resin was washed with DMF $(4 \times 3 \mathrm{~mL}$ for $30 \mathrm{~s}), 20 \% v / v$ piperidine in NMP $(3 \times 3 \mathrm{~mL}$ for $3 \mathrm{~min})$, NMP $(3 \times 3 \mathrm{~mL}$ for $30 \mathrm{~s})$, dichloromethane $(3 \times 5 \mathrm{~mL}$ for $30 \mathrm{~s})$ and NMP $(3 \times 3 \mathrm{~mL}$ for $30 \mathrm{~s})$.

Important notes (1): it is critical to limit to $5 \mathrm{~min}$ the incubation with the aldehyde, to keep subsequent washes as short as possible and to add without delay the $\mathrm{NaBH}_{4}$ solution after washes. The Prelude synthesizer that we use operates its six reaction vessels (RV) in a successive fashion rather than in parallel. We therefore prefer to program the reductive amination protocol one RV after the other. 
Important notes (2): "fresh" solutions of $\mathrm{NaBH}_{4}$ can be prepared up to at least $24 \mathrm{~h}$ in advance before use without any loss of efficiency. Use peptide-grade DMF for all three reductive amination steps.

Coupling of the C-terminal amino acid of the peptide crypto-thioester segment sequence: as disclosed in our previous works, the $\mathrm{N}$-acylation kinetics highly depends on the C-terminal residue. If non-hindered aminoacids can be easily introduced through standard Fmoc-SPPS protocols, beta-substituted ones (Ile, Val, Thr) and Pro require prolonged coupling (slow $O$-to- $N$ acyl shift ${ }^{[17 a]}$ ) and epimerization-prone residue (Cys/His) needs particular attention.

For Ala / Arg / Asn / Gln / Gly / Leu / Lys / Met / Phe / Ser / Trp / Tyr: protected amino acids $(0.25 \mathrm{mmol}, 10$ equiv.) were coupled using HCTU (98 mg, $0.238 \mathrm{mmol}, 9.5$ equiv.) and DIEA ( $87 \mu \mathrm{L}, 0.5 \mathrm{mmol}, 20$ equiv.) in NMP $(2.5 \mathrm{~mL})$ for $3 \times 3 \mathrm{~h}$. A single $30 \mathrm{~min}$ coupling is sufficient for Gly and Ala,${ }^{[110]}$ but we prefer to routinely use this long triple coupling protocol for synthesizer programming simplicity.

For lle / Pro / Thr / Val: protected amino acids ( $0.25 \mathrm{mmol}, 10$ equiv.) were

For epimerization-prone amino acids Cys / His: protected amino acids $(0.25 \mathrm{mmol}, 10$ equiv.) were coupled using DIC (39 $\mu \mathrm{L}, 0.25 \mathrm{mmol}, 10$ equiv.) and oxyma (45 mg, $0.075 \mathrm{mmol}, 10$ equiv.) in NMP (2.5 mL) for 12 h.

Note that unprotected carboxylic acid side chains of Asp and Glu are known to react intramolecularly with the $\mathrm{C}^{\alpha}$ thioester function leading to $\mathrm{C}$ terminal anhydrides and thus the obtention of isoAsp and isoGlucontaining ligation product, respectively, $[9 e, 49]$ and we do not recommend the use of Asp- and Glu- $\mathrm{N}$-Hnb-Cys crypto-thioesters. We observed this deleterious side-reaction for Asp, and did not check it for Glu.

In all cases, we routinely perform a capping step of eventual unreacted amine groups by four consecutive treatments with acetic anhydride (143 $\mu \mathrm{L}, 1.51 \mathrm{mmol}, 60$ equiv), $\mathbb{P r}_{2} \mathrm{NEt} \quad(68 \mu \mathrm{L}, 0.39 \mathrm{mmol}, 15.5$ equiv.) and $\mathrm{HOBt}$ (6 mg, $0.044 \mathrm{mmol}, 1.8$ equiv.) in NMP (3 mL) for $7 \mathrm{~min}$.

Crypto-thioester peptide elongation: classical Fmoc-SPPS elongation using uronium coupling agents (HBTU/HOBt, HCTU, HATU) should be preferred. In our hands, this gave similar results in terms of qualitative HPLC chromatogram as for peptide segments not incorporating a Cterminal $\mathrm{N}$-Hnb-Cys device.

Important note (3): phosphonium coupling reagents should be avoided, as we observed side-reaction corresponding to $O-P$ bond formation on the Hnb phenol group when using PyAOP or PyBOP.

Important note (4): microwave- and other heat-accelerated synthesis of $\mathrm{N}$-Hnb-Cys crypto-thioesters probably remains to be optimized, as byproducts arising from $\beta$-elimination of the $\mathrm{Cys}(\mathrm{StBu})$ followed by Michaeltype addition of piperidine were observed. Note that we detected small amounts of this by-product at room temperature for a very long peptide (compound S24, see supporting information, p S47-S48), but this sidereaction is probably sequence-dependant: indeed, a study on a short model peptide did not show any trace of by-product even after a very long piperidine treatment (supporting information, p S48-S49).

Important note (5): variable amount (5-90\%) of O-acylation of the $\mathrm{Hnb}$ phenol are observed after each coupling. This ester is cleaved within seconds upon piperidine treatment during Fmoc deprotection. As we make use of 10 equiv. of Fmoc-Xaa-OH for each couplings, that is not a problem in terms of elongation yields. As a consequence, a final piperidine treatment (standard $3 \times 3 \mathrm{~min}, 20 \%$ in DMF or NMP) is thus necessary even if the N-terminal residue is not Fmoc-protected (Boc, acetyl, biotin etc...). ${ }^{[18]}$ Moreover, UV titration of the fluorenylmethyl-piperidine adduct after Fmoc deprotection is useless unless using a prior treatment with hydroxylamine/imidazole ${ }^{[50]}$ for selective ester cleavage before piperidine treatment (supporting information, p S3).

\section{Acknowledgements}

We thank Dr Guillaume Gabant, Dr Cyril Colas and the mass spectrometry platforms of CBM and FR2708 federation for the MS and LC/HRMS analyses, as well as Hervé Meudal and the CBM nuclear magnetic resonance platform for assistance in the recording of NMR spectra and their analyses. We also warmly thank Jean-Baptiste Madinier for his assistance in automated peptide synthesis, and Agnès Delmas for proofreading the manuscript. University of Orléans and doctoral school SSBCV (ED549) are gratefully acknowledged for a $\mathrm{PhD}$ fellowship for SAA, as well as French national funding agency ANR (EasyMiniprot project, ANR-15-CE07-0022) for financial support.

\section{References and Notes}

[1] a) P. Dawson, T. Muir, I. Clark-Lewis, S. Kent, Science 1994, 266, 776 779; b) V. Agouridas, O. El Mahdi, V. Diemer, M. Cargoët, J.-C. M Monbaliu, O. Melnyk, Chem. Rev. 2019, 119, 7328-7443.

[2] Note that Pentelute and his co-workers recently reported a flow-based approach for the synthesis of polypeptides of more than 200 aa See for example: N. Hartrampf, A. Saebi, M. Poskus, Z. P. Gates, A. J. Callahan, A. E. Cowfer, S. Hanna, S. Antilla, C. K. Schissel, A. J. Quartararo, X. Ye, A. J. Mijalis, M. D. Simon, A. Loas, S. Liu, C. Jessen, T. E. Nielsen, B. L. Pentelute, Science 2020, 368, 980-987.

[3] For a database dedicated to protein chemical synthesis, see: V Agouridas, O. El Mahdi, M. Cargoët, O. Melnyk, Bioorg. Med. Chem. 2017, 25, 4938-4945.

[4] See for example: a) M. T. Weinstock, M. T. Jacobsen, M. S. Kay, Proc Natl. Acad. Sci. USA 2014, 111, 11679-11684; b) A. Pech, J. Achenbach, M. Jahnz, S. Schülzchen, F. Jarosch, F. Bordusa, S. Klussmann, Nucleic Acids Res. 2017, 45, 3997-4005; c) J. Weidmann, M. Schnölzer, P. E. Dawson, J. D. Hoheisel, Cell Chem. Biol. 2019, 26, 645-651; d) M. Wang, W. Jiang, X. Liu, J. Wang, B. Zhang, C. Fan, L. Liu, G. Peña-Alcántara J.-J. Ling, J. Chen, T. F. Zhu, Chem. 2019, 5, 848-857; e) H. Sun, A Brik, Acc. Chem. Res. 2019, 52, 12, 3361-3371.

[5] a) F. Mende, O. Seitz, Angew. Chem. Int. Ed. 2011, 50, 1232-1240; b) H. Li, S. Dong, Sci. China Chem. 2017, 60, 201-213.

[6] G.-M. Fang, Y.-M. Li, F. Shen, Y.-C. Huang, J.-B. Li, Y. Lin, H.-K. Cui, L. Liu, Angew. Chem. Int. Ed. 2011, 50, 7645-7649.

[7] (a) J. B. Blanco-Canosa, P. E. Dawson, Angew. Chem. Int. Ed. 2008, 47 6851-6855; (b) J. B. Blanco-Canosa, B. Nardone, F. Albericio, P. E. Dawson, J. Am. Chem. Soc. 2015, 137, 7197-7209.

[8] E. C. B. Johnson, S. B. H. Kent, J. Am. Chem. Soc. 2006, 128, 66406646

[9] For peptide hydrazides, see for example: a) P. Siman, S. V. Karthikeyan, M. Nikolov, W. Fischle, A. Brik, Angew. Chem. Int. Ed. 2013, 52, 80598063; b) S.K. Mong, A.A. Vinogradov, M.D. Simon, B.L. Pentelute, ChemBioChem 2014, 15, 721-733; c) X. Tian, J. Li, W. Huang Tetrahedron Lett. 2016, 57, 4264-4267; For Nbz, see for example: d) S. K. Mahto, C. J. Howard, J. C. Shimko, J. J. Ottesen, Chembiochem 2011 12, 2488-2494; e) J. Mannuthodikayil, S. Singh, A. Biswas, A. Kar, W Tabassum, P. Vydyam, M. K. Bhattacharyya, K. Mandal, Org. Lett. 2019 21, 9040-9044.

[10] For recent examples, see: a) H. E. Elashal, Y. E. Sim, M. Raj, Chem. Sci 2017, 8, 117-123; b) D. T. Flood, J. C. J. Hintzen, M. J. Bird, P. A Cistrone, J. S. Chen, P. E. Dawson, Angew. Chem. Int. Ed. 2018, 57 11634-11639.

[11] See for examples: a) T. Kawakami, M. Sumida, K. Nakamura, T. Vorherr S. Aimoto, Tetrahedron Lett. 2005, 46, 8805-8807; b) N. Ollivier, J. B. Behr, O. El-Mahdi, A. Blanpain, O. Melnyk, Org. Lett. 2005, 7, 26472650; c) Nagaike, F., Onuma, Y., Kanazawa, C., Hojo, H., Ueki, A., Nakahara, Y., Nakahara, Y. Org. Lett. 2006, 8, 4465-4468; d) Y. Ohta 
S. Itoh, A. Shigenaga, S. Shintaku, N. Fujii, A. Otaka, Org. Lett. 2006, 8, 467-470; e) H. Hojo, Y. Onuma, Y. Akimoto, Y. Nakahara, Y. Nakahara Tetrahedron Lett. 2007, 48, 25-28; f) Kawakami, T., Aimoto, S. Chem. Lett. 2007, 36, 76-77; g) J. Kang, J. P. Richardson, D. Macmillan, Chem Commun. 2009, 407-409; h) S. Tsuda, A. Shigenaga, K. Bando, A Otaka, Org. Lett. 2009, 11, 823-826; i) N. Ollivier, J. Dheur, R. Mhidia A. Blanpain, O. Melnyk, Org. Lett. 2010, 12, 5238-5241; j) W. Hou, X Zhang, F. Li, C.-F. Liu, Org. Lett. 2011, 13, 386-389; k) K. Sato, A Shigenaga, K. Tsuji, S. Tsuda, Y. Sumikawa, K. Sakamoto, A. Otaka, ChemBioChem 2011, 12, 1840-1844; I) R. Sharma, J. P. Tam, Org. Lett 2011, 13, 5176-5179; m) J. S. Zheng, H. N. Chang, F. L. Wang, L. Liu, J. Am. Chem. Soc. 2011, 133, 11080-11083; n) F. Burlina, G. Papageorgiou, C. Morris, P. D. White, J. Offer, Chem. Sci. 2014, 5, 766770; o) Y. Asahina, K. Nabeshima, H. Hojo, Tetrahedron Lett., 2015, 56 1370-1373; p) V. P. Terrier, H. Adihou, M. Arnould, A. F. Delmas, V. Aucagne, Chem. Sci. 2016, 7, 339-345; q) P. M. M. Shelton, C. E. Weller C. Chatterjee, J. Am. Chem. Soc. 2017, 139, 3946-3949; r) M. Yanase, K. Nakatsu, C. J. Cardos, Y. Konda, G. Hayashi, A. Okamoto, Chem. Sci. 2019, 10, 5967-5975; s) K. Nakatsu, M. Yanase, G. Hayashi, A.

Nishiuchi, A. Otaka, J. Org. Chem. 2012, 77, 6948-6958.

[13] Y. Asahina, H. Hojo, J. Org. Chem. 2020, 85, 1458-1465.

[14] C. A. Hunter, Angew. Chem. Int. Ed. 2004, 43, 5310-5324.

[15] Note that DFT calculations on $\mathrm{N}$-Hnb-Cys mechanism were undertaken by others, see: S. Bi, P. Liu, B. Ling, X. Yuan, Y. Jiang, Chin. Chem. Lett. 2018, 29, 1264-1268.

[16] a) T. Johnson, M. Quibell, D. Owen, R.C. Sheppard, J. Chem. Soc., Chem. Commun. 1993, 369-372. b) L. P. Miranda, W. D. Meutermans, M. L. Smythe, P. F. Alewood, J. Org. Chem. 2000, 65, 5460-5468.

[17] a) D. Lelièvre, V. P. Terrier, A. F. Delmas, V. Aucagne, Org. Lett. 2016 18, 920-923; b) G. Martinez, J.-P. Hograindleur, S. Voisin, R. Abi Nahed, T. M. Abd El Aziz, J. Escoffier, J. Bessonnat, C.-M. Fovet, M. De Waard S. Hennebicq, V. Aucagne, P. F. Ray, E. Schmitt, P. Bulet, C. Arnoult, Mol. Hum. Reprod. 2016, 10,116-131; c) V. P. Terrier, A. F. Delmas, V. Aucagne, Org. Biomol. Chem. 2017, 15, 316-319; d) K. Loth, A. Vergnes C. Barreto, S. N. Voisin, H. Meudal, J. Da Silva, A. Bressan, N. Belmadi, E. Bachère, V. Aucagne, C. Cazevielle, H. Marchandin, R. D. Rosa, P. Bulet, L. Touqui, A. F. Delmas, D. Destoumieux-Garzón, mBio 2019, 10, e01821-19.

[18] L. De Rosa, R. Di Stasi, L. D. D’Andrea, Tetrahedron 2019, 75, 894-905.

[19] H. Rink, Tetrahedron Lett. 1987, 28, 3787-3790.

[20] Note that we tried different other conditions found in the literature for solid-supported reductive aminations, involving either a one pot process or two consecutive steps, but with inferior results, resulting in either uncomplete reaction, bis-alkylation or to other unidentified byproducts. See reference [110].

[21] F. García-Martín, M. Quintanar-Audelo, Y. García-Ramos, L. J. Cruz, C. Gravel, R. Furic, S. Côté, J. Tulla-Puche, F. Albericio, J. Comb. Chem. 2006, 8, 213-220.

[23] In our seminal study, a notable side-product was only observed when introducing a Cys(Trt) as the first amino-acid of the peptide segment, which we attributed to epimerization considering the know propensity of Cys to epimerize during coupling (see reference 28). We also observed an expected co-product after coupling of Asn, due to incomplete deprotection of the $N$-Trt group (see: M. Friede, S. Denery, J. Neimark, S. Kieffer, H. Gausepohl, J. P. Briand, Pept. Res. 1992, 5, 145-147).

[24] a) A. Czarnik, Biotechnol. Bioeng. 1998, 61, 77-79. b) Y. Feng, K. Burgess, Biotechnol. Bioeng. 2000, 71, 3-8.

[25] https://spheritech.com/spheritide-Ks/.

[26] J.H. Adams, R.M. Cook, D. Hudson, V. Jammalamadaka, M.H. Lyttle, M.F. Songster, J. Org. Chem. 1998, 63, 3706-3716.

[27] See for example: a) N. Robertson, L. Jiang, R. Ramage, Tetrahedron 1999, 55, 2713-2720. b) W. Van Den Nest, S. Yuval, F. Albericio, J. Pept. Sci. 2001, 7, 115-120. c) Y.E. Jad, S.N. Khattab, B.G. de la Torre, T. Govender, H.G. Kruger, A. El-Faham, F. Albericio, Org. Biomol. Chem. 2014, 12, 8379-8385.

[28] See for example: a) T. Kaiser, G. J. Nicholson, H. J. Kohlbau, W. Voelter, Tetrahedron Lett. 1996, 37, 1187-1190; b) Y. Han, F. Albericio, G. Barany, J. Org. Chem. 1997, 62, 4307-4312.

[29] See for example: a) H. Hibino, Y. Nishiuchi, Tetrahedron Lett. 2011, 52, 4947-4949.
[30] Note that in our previously reported work, a model peptide generated using this device was proven to be epimerization-free after NCL: see reference [110]

[31] R. Huisgen, Angew. Chem. 1955, 67, 360-360.

[32] a) D. A. Jaeger, D. J. Cram, J. Am. Chem. Soc. 1971, 93, 5153-5161; b) T. F. Buckley, H. Rapoport, J. Am. Chem. Soc. 1982, 104, 4446-4450 c) G. Cainelli, D. Giacomini, A. Trerè, P. P. Boyl, J. Org. Chem. 1996, 61, 5134-5139

[33] C. G. Boojamra, K. M. Burow, L. A. Thompson, J. A. Ellman, J. Org Chem. 1997, 62, 1240-1256.

[34] M. Petrisko, J. Krupka, Res. Chem. Intermed. 2005, 31, 769-778.

[35] A. Lawson, J. O. Stevens, J. Chem. Soc. 1968, 1514-1515.

[36] See for example: a) H. B. Dixon, V. Moret, Biochem J. 1965, 94, 463469; b) H. B. Dixon, R. Fields, Methods Enzymol. 1972, 25, 409-419 c) T. Hara, A. Tainosho, K. Nakamura, T. Sato, T. Kawakami, S. Aimoto. J Pept. Sci. 2009, 15, 369-376.

[37] E. W. Baxter, A. B. Reitz, in Organic Reactions, Vol. 59, John Wiley \& Sons, Inc., Hoboken, NJ, USA, 2002, pp. 1-714.

[38] Note that we also tested the use of Lewis acids in combination with unidentified byproducts. See supporting information, table S1, entries 1 , 6 and 8.

[39] Recording of the NMR spectra at higher temperatures did not afford a single peak set, but rather a massive loss of resolution, consistent with slow conformational exchange in the NMR timescale.

[40] In the case of the $\mathbf{1 0} \mathbf{c} / \mathbf{1 0 d}$ mixture, available in larger quantity than pure 10a-c, we were able to record a ${ }^{1} \mathrm{H}-{ }^{13} \mathrm{C} \mathrm{HMBC}$ spectrum which confirmed that the newly appeared $\mathrm{CH}$ signals were coupled to carbon signals of the phenol ring, see supporting information $p$ S41.

[41] a) P. Gomes, M. J. Araújo, M. Rodrigues, N. Vale, Z. Azevedo, J. lley, P. Chambel, J. Morais, R. Moreira, Tetrahedron 2004, 60, 5551-5562; b) A L. Satz, J. Cai, Y. Chen, R. Goodnow, F. Gruber, A. Kowalczyk, A Petersen, G. Naderi-Oboodi, L. Orzechowski, Q. Strebel, Bioconjugate Chem. 2015, 26, 1623-1632; c) Y. Wang, A.Ø. Madsen, F. Diness, M. Meldal, Chem. Eur. J. 2017, 23, 13869-13874.

[42] W. P. Unsworth, G. Coulthard, C. Kitsiou, R. J. K. Taylor, J. Org. Chem 2014, 79, 1368-1376.

[43] a) V. Adebomi, R. D. Cohen, R. Wills, H. A. H. Chavers, G. E. Martin, M Raj, Angew. Chem. Int. Ed. 2019, 58, 19073-19080. b) B. Koo, N. S. Dolan, K. Wucherer, H. K. Munch, M. B. Francis, Biomacromolecules 2019, 20, 3933-3939.

[44] Relative amounts of 10a-d depend on the reaction conditions (solvent, additive, reducing agent etc.), probably because of variable amounts of Cys epimerization and low diastereoselectivity of the cyclization.

[45] ${ }^{1} \mathrm{H}$ NOESY and ROESY analyses were performed to try to determine distereoisomers configurations, however signals overlap did not allow stereochemical attribution.

[46] Note that we also tested the use of Lewis acids in combination with $\mathrm{NaBH}_{4}$, and tried alternative reducing agents, but this led either to a greater amount of epimerization, or the formation of other byproducts. See supporting information, table S1.

[47] See for example: a) V. Aucagne, I. E. Valverde, P. Marceau, M. Galibert N. Dendane, A. F. Delmas, Angew. Chem. Int. Ed. 2012, 51, 1132011324; b) I. E. Decostaire, D. Lelièvre, V. Aucagne, A. F. Delmas, Org. Biomol. Chem. 2014, 12, 5536-5543; c) M. Galibert, V. Piller, F. Piller, V. Aucagne, A. F. Delmas, Chem. Sci. 2015, 6, 3617-3623.

[48] For recent examples of solid-supported aminations of peptides, see: a) A.-B. M. Abdel-Aal, G. Papageorgiou, M. Quibell, J. Offer, Chem. Commun. 2014, 50, 8316-8319; b) K. Pels, T. Kodadek, ACS Comb. Sci. 2015, 17, 152-155; c) A.-B. M. Abdel-Aal, G. Papageorgiou, R. Raz, M. Quibell, F. Burlina, J. Offer, J. Pept. Sci. 2016, 22, 360-367; d) S. F. Loibl, A. Dallmann, K. Hennig, C. Juds, O. Seitz, Chem. Eur. J. 2018, 24, 3623-3633. e) F. Burlina, A.-B. M Abdel-Aal, R. Raz, I. Pinzuti, G. Papageorgiou, J. Li, R. Antrobus, S. R. Martin, S. Kunzelmann, B. Stieglitz, J. Offer, Commun. Chem, 2019, 2, 111.

[49] See for example: a) M. Jbara, E. Eid, A. Brik, Org. Biomol. Chem. 2018 16, 4061-4064; b) N. Kamo, G. Hayashi, A. Okamoto, Chem. Commun. 2018, 54, 4337-4340.

[50] J. J. Díaz-Mochón, L. Bialy, M. Bradley, Org. Lett. 2004, 6, 1127-1129. 